\title{
Nodal Signals through Activin Receptor-Like Kinase 7 to Inhibit Trophoblast Migration and Invasion
}

\author{
Implication in the Pathogenesis of Preeclampsia
}

\author{
Lubna Nadeem, ${ }^{*}$ Sadia Munir,, Guodong Fu, ${ }^{*}$ \\ Caroline Dunk, ${ }^{\dagger}$ Dora Baczyk, ${ }^{\dagger}$ \\ Isabella Caniggia, ${ }^{\dagger \ddagger}$ Stephen Lye,$^{\dagger \neq}$ \\ and Chun Peng* \\ From the Department of Biology, "York University, Toronto; the \\ Research Centre for Women's and Infants Health, ${ }^{\dagger}$ Samuel \\ Lunenfeld Research Institute, Mount Sinai Hospital, Toronto; and \\ the Departments of Physiology and Obstetrics and Gynecology, \\ The University of Toronto, Toronto, Ontario, Canada
}

Trophoblast cell invasion into the uterus is an essential process for successful pregnancy, and shallow invasion of trophoblasts into the maternal decidua is linked to preeclampsia. We have reported that Nodal, a member of the transforming growth factor- $\beta$ superfamily, acts through activin receptor-like kinase 7 (ALK7) to inhibit trophoblast proliferation and to induce apoptosis. In this study, we examined the spatial and temporal expression patterns of Nodal and ALK7 in human placenta from normal and preeclamptic pregnancies and investigated whether Nodal regulated trophoblast migration and invasion. Nodal and ALK7 were detected in villous and extravillous trophoblast cell populations in early gestation, and their levels were strongly up-regulated in preeclamptic placenta. Overexpression of Nodal or constitutively active ALK7 decreased cell migration and invasion, whereas knockdown of Nodal and ALK7 had the opposite effects. In placental explant culture, treatment with Nodal inhibited trophoblast outgrowth, whereas Nodal small-interfering RNA strongly induced the expansion of explants and the migration of extravillous trophoblast cells. Nodal stimulated the secretion of tissue inhibitor of metalloproteinase-1 and inhibited matrix metalloproteinase (MMP)-2 and MMP-9 activity. These findings suggest that the Nodal/ALK7 pathway plays important roles in human placentation and that its abnormal signaling may contribute to the development of preeclampsia. (Am J Pathol 2011, 178:1177-1189; DOI: 10.1016/j.ajpath.2010.11.066)
Nodal is a member of the transforming growth factor- $\beta$ superfamily. ${ }^{1}$ Similar to other members of this superfamily, Nodal signals through type II and type I serine/threonine kinase receptors present on the cell membrane. Specifically, Nodal has been reported to act through two type II receptors (activin receptors $\| \mathrm{A}$ and $\mathrm{IB}$ ) and two type I receptors (activin receptor-like kinases 4 and 7 ) (ALK4 and ALK7). ${ }^{2}$ In addition, Cripto, a member of the EGF-CFC family of proteins, has been shown to act as a coreceptor for Nodal and enhances Nodal signaling via ALK4. ${ }^{3}$

Nodal was first discovered and cloned from a mouse embryo cDNA library, ${ }^{1}$ and its role in the establishment of left-right and anteroposterior axes patterning and the induction of dorsal mesoderm is well established. ${ }^{4-7} \mathrm{Al}-$ though the role of Nodal in extraembryonic tissues is still vague, several studies have suggested that Nodal is important for placental development. A hypomorphic mutation of the nodal gene causes abnormal placental development, including expansion of giant cells and spongiotrophoblast layers and a decrease in labyrinthine development. ${ }^{8}$ Nodal also acts on extraembryonic stem cells to inhibit precocious differentiation of trophoblast stem cells. ${ }^{9}$ In the human placenta, expression of Nodal and ALK7 has previously been reported. ${ }^{10}$ We demonstrated that Nodal, signaling through ALK7, inhibits trophoblast proliferation and induces apoptosis. ${ }^{11}$ These findings suggest that the Nodal/ALK7 pathway is a key player in the regulation of trophoblast differentiation, placental development, and function.

The extent of human trophoblast invasion into the uterus is precisely controlled by maternal and tropho-

Supported by grants from the Canadian Institutes of Health Research (MOP-81370 to C.P.; MOP-82811 to S.L.); by an Ontario Graduate Scholarship (L.N.); and by a Mid-Career Award from the Ontario Women's Health Council/Canadian Institutes of Health Research (C.P. and I.C.).

Accepted for publication November 18, 2010.

Address reprint requests to Chun Peng, Ph.D., Department of Biology, York University, 4700 Keele St, Toronto, ON M3J 1P3, Canada. E-mail: cpeng@yorku.ca. 
blast-derived factors, including cytokines, growth factors, and hormones. ${ }^{12-14}$ In early placental development, the trophoblast presents a heterogeneous population of cells that differentiates into two different lineages: extravillous trophoblasts (EVTs; anchoring villi) and cytotrophoblasts (CTBs; floating villi). In floating villi, CTBs remain attached to the basement membrane as a monolayer of proliferating polarized cells, which differentiate and fuse to form the nonproliferative multinucleated syncytiotrophoblast (STB) layer. In anchoring villi, however, CTBs outgrow the STB layer and form CTB columns of nonpolarized invasive cells. From the distal ends of CTB columns, EVTs that are nonproliferative yet exhibit highly invasive behavior detach and invade the maternal decidua. In the uterus, EVTs target and transform the spiral arteries, leading to increased blood flow at the uteroplacental interface. ${ }^{15}$ Failure of EVT invasion can lead to intrauterine growth restriction and preeclampsia. ${ }^{16,17}$

Preeclampsia, a pregnancy-specific disorder characterized by hypertension and proteinuria, is a major cause of maternal and fetal morbidity and mortality. ${ }^{18}$ Defects in placentation, particularly trophoblast invasion, are thought to contribute to the onset of this disorder. It is believed that shallow invasion of EVTs into the uterus leads to insufficient transformation of the myometrial spiral arteries, resulting in inadequate blood supply and causing oxidative stress in the placenta. ${ }^{19}$ Several ligands and receptors in the transforming growth factor- $\beta$ superfamily have been implicated in the development of preeclampsia. ${ }^{20-23}$ Interestingly, a type $\|$ receptor (activin receptor IIA) and two type I receptors (ALK2 and ALK7) are located on chromosome 2q22, which contains a preeclampsia susceptibility locus, and the potential association of these receptors, particularly activin receptor IIA, with preeclampsia has been suggested. ${ }^{24,25}$

To further elucidate the role of Nodal/ALK7 signaling in human placenta, we performed immunohistochemical $(\mathrm{IHC})$ analysis to determine Nodal and ALK7 expression in normal and preeclamptic placentae. We also investigated the role and mechanism of Nodal in trophoblast cell migration and invasion. The present findings suggest that Nodal and ALK7 play an important role in regulating trophoblast migration and invasion and that their abnormal signaling may contribute to the development of preeclampsia.

Table 1. Clinical Data for Preeclamptic Patients and Healthy Pregnant Women

\begin{tabular}{lcr}
\hline & $\begin{array}{c}\text { Preterm control } \\
(n=5)\end{array}$ & $\begin{array}{c}\text { Preeclamptic } \\
(n=8)\end{array}$ \\
\hline Gestational age (weeks) & $32.8 \pm 0.83$ & $29.8 \pm 2.03$ \\
SBP (mmHg) & $126.2 \pm 3.11$ & $169.3 \pm 13.1$ \\
DBP (mmHg) & $74.4 \pm 4.15$ & $106.1 \pm 9.81$ \\
Proteinuria & 0 & $3.71 \pm 0.48$ \\
Parity & $1.6 \pm 0.54$ & $0.87 \pm 1.35$ \\
Gravidity & $0.2 \pm 0.44$ & $2.25 \pm 1.28$ \\
\hline
\end{tabular}

Data are given as mean $\pm \mathrm{SD}$.

SBP, systolic blood pressure; DBP, diastolic blood pressure.

\section{Materials and Methods}

\section{Patients and Placental Tissue Collection}

Human placentae were collected at the Department of Obstetrics and Gynecology, Mount Sinai Hospital, and at the Morgantaler Clinic, Toronto, ON, Canada. First- and second-trimester placentae were obtained from patients undergoing terminated pregnancies via a dilation and curettage procedure, and third-trimester placentae were from abdominal delivery or cesarean delivery. The collection and use of placental samples is approved by the Mount Sinai Hospital Research Ethics Board, and all the subjects provided written informed consent. Fifty-one placentae were used in this study. Among them, firsttrimester ( 5 to 12 weeks, $n=13$ ), second-trimester (13 to 19 weeks, $n=8$ ), and term (33 to 40 weeks, $n=9$ ) were used to examine the expression profile of Nodal and ALK7 during different stages of pregnancy. To determine whether there is abnormal expression of Nodal and/or ALK7 in preeclamptic placenta, eight placentae taken from patients with severe preeclampsia and five preterm controls were used for $\mathrm{IHC}$ studies. Preterm tissues were collected from laboring cesarean deliveries after fetal distress or from premature rupture of membrane spontaneous labor. All cases of preterm tissues were reviewed by a placental pathologist, and no gross abnormalities or signs of chorioamnionitis were observed. Clinical data for the preeclamptic patients and the preterm controls are summarized in Table 1. Eight first-trimester placentae (6 to 9 weeks) were used for explant culture.

\section{Cell Line and Cell Culture}

An immortalized human trophoblast cell line, HTR-8/ SVneo, established from first-trimester human CTB cells, was obtained from Dr. Charles Graham (Queen's University, Kingston, ON, Canada) and was cultured and maintained as described previously. ${ }^{26}$ Briefly, cells were cultured in RPMI 1640 medium supplemented with 10\% fetal bovine serum, $100 \mathrm{IU} / \mathrm{ml}$ of penicillin, and $100 \mu \mathrm{g} / \mathrm{ml}$ of streptomycin at $37^{\circ} \mathrm{C}$ in a humidified atmosphere of $5 \%$ $\mathrm{CO}_{2}$. All reagents and culture media were purchased from Invitrogen Canada Inc. (Burlington, ON, Canada).

\section{First-Trimester Human Placental Explant Culture}

Explant cultures were performed as previously described. ${ }^{27}$ Small villous explants were dissected and placed on Transwell inserts (Millipore, Billerica, MA) precoated with $200 \mu \mathrm{L}$ of undiluted phenol red-free Matrigel (BD Biosciences, Bedford, MA) in a 24-well culture plate. Explants were allowed to attach to the Matrigel overnight and then were supplied with serum-free culture media supplemented with $100 \mathrm{U} / \mathrm{ml}$ of penicillin, $100 \mathrm{U} / \mathrm{ml}$ of streptomycin, $2 \mathrm{mmol} / \mathrm{L}$ L-glutamine, $100 \mu \mathrm{g} / \mathrm{ml}$ of gentamicin, and $2.5 \mu \mathrm{g} / \mathrm{ml}$ of fungizone and incubated at $37^{\circ} \mathrm{C}$ with $3 \% \mathrm{O}_{2}$ and $5 \% \mathrm{CO}_{2}$ as described earlier. ${ }^{28}$ After 2 days of culture establishment, all the villous tips 
Table 2. List of siRNAs Used in This Study

\begin{tabular}{|c|c|c|}
\hline Target mRNA & Primer & Sequence \\
\hline \multirow[t]{2}{*}{ ALK7 siRNA } & Sense & $5^{\prime}$-CCAAGAGGUAUAUGGCUCCt $t-3^{\prime}$ \\
\hline & Antisense & $5^{\prime}$-GGAGCCAUAUACCUCUUGGtt $-3^{\prime}$ \\
\hline \multirow[t]{2}{*}{ ALK4 SiRNA } & Sense & $5^{\prime}$-CUACACGUGUGAGACAGAUtt $-3{ }^{\prime}$ \\
\hline & Antisense & $5^{\prime}$-AUCUGUCUCACACGUGUAGtt $-3^{\prime}$ \\
\hline \multirow[t]{2}{*}{ Nodal siRNA } & Sense & $5^{\prime}$-AGACAUGAUCGUGGAAGAAtt $-3^{\prime}$ \\
\hline & Antisense & $5^{\prime}$-UUCUUCCACGAUCAUGUCUtt- $3^{\prime}$ \\
\hline \multirow{2}{*}{ Control siRNA } & Sense & $5^{\prime}$-UUCUCCGAACGUGUCACGUtt-3' \\
\hline & Antisense & $5^{\prime}$-ACGUGACACGUUCGGAGAAtt $-3^{\prime}$ \\
\hline
\end{tabular}

Lowercase t represents deoxythymidine.

were carefully observed under the dissecting microscope, and only those with successful EVT outgrowths were selected for treatments with recombinant Nodal (rNodal) (R\&D Systems, Minneapolis, MN), $250 \mathrm{ng} / \mathrm{ml}$, or Nodal small-interfering RNA (siRNA) (see Table 2 for siRNA sequences). All siRNAs were synthesized by GenePharma Co. (Shanghai, China). Explants were photographed before and after treatment using a Leica DFC400 camera (Leica Microsystems GmbH, Wetzlar, Germany) attached to a dissecting microscope, and explant outgrowths were measured using ImageJ software. Area of outgrowth was determined by subtracting the total outgrowth area at the end of the experiment (72 hours after treatment) from that of the initial area before treatment. Each treatment was performed in triplicate, and each experiment was repeated three times using different placentae.

\section{IHC Analysis}

Placental tissues embedded in paraffin were obtained from the Research Centre for Women's and Infant's Health Biobank at Mount Sinai Hospital. Explant tissues attached to Matrigel were collected and washed with cold $1 \times$ PBS and fixed with $4 \%$ paraformaldehyde for 1 hour. Tissues were dehydrated in ascending concentrations of ethanol, cleared in xylene, and embedded in paraffin. Tissue sections $5 \mu \mathrm{m}$ thick were prepared, and every third serial section was histologically assessed by means of standard H\&E staining. An IHC analysis was performed using the streptavidin peroxidase method as previously described. ${ }^{29}$ Briefly, the paraffin-embedded tissue sections were dewaxed using xylene and were rehydrated in descending grades of ethanol. Sections were incubated in 3\% hydrogen peroxide in methanol solution for 30 minutes to quench the endogenous peroxidase activity and then were subjected to antigen retrieval by heating the slides in a microwave oven in 10 $\mathrm{mmol} / \mathrm{L}$ citrate buffer. After cooling to room temperature, the sections were washed twice with $1 \times$ PBS. Blocking was performed in a humidified chamber with Dako blocking serum (Dako Diagnostics Canada Inc., Mississauga, ON, Canada) for 1 hour at room temperature. Sections were then incubated with a primary antibody overnight at $4^{\circ} \mathrm{C}$, washed, and incubated with a secondary antibody for 1 hour. After incubation with streptavidin-labeled horseradish-peroxidase-conjugated antibody (Dako), slides were developed using a Dako DAB staining kit containing $0.02 \% \mathrm{H}_{2} \mathrm{O}_{2}$ and $0.075 \% 3,3^{\prime}$-diaminobenzi- dine in $1 \times$ PBS. Counterstaining was performed using Harris hematoxylin (1:2; Sigma-Aldrich Corp., St. Louis, $\mathrm{MO})$, and sections were dehydrated in ascending series of ethanol, cleared in xylene, and mounted with Cytoseal (Fisher Scientific, Ottawa, ON, Canada). Photographs were taken using a Nikon Coolpix 4500 camera attached to a Nikon Light Microscope Eclipse 55i (Nikon Canada Inc., Mississauga, ON, Canada). Antibodies used for IHC analysis were HLA-G (1:100; Abcam, Cambridge, England), Nodal (1:100; Santa Cruz Biotechnology, Santa Cruz, CA), and ALK7 (1:50; R\&D Systems). Biotinylated secondary antibodies against mouse, rabbit, and goat were used (1:300; Sigma-Aldrich Corp.). Negative controls were performed by omitting the primary antibodies and using their respective IgGs. The intensity of $\mathrm{IHC}$ staining for Nodal and ALK7 was scored on blinded samples using a scale of increasing intensity: -, no staining; + , weak staining; ++ , moderate staining; +++ , strong staining; and ++++ , strongest staining. Tissues that received a score of $+/-$ indicated that in some of the samples, no signal was detected and in others, weak signal was observed.

\section{Wound-Healing Assays}

Cells were cultured in 6-well plates and were transfected with $4 \mu \mathrm{g}$ of plasmid DNA of pcDNA4 (empty vector control), constitutively active ALK7 (ALK7-ca), or Nodal in triplicate. For visual monitoring of transfected cells, cotransfection was performed using plasmid encoding enhanced green fluorescent protein (Bioscience Clontech, Mississuaga, ON, Canada). Transient transfection was performed using Lipofectamine 2000 (Invitrogen). Generation of expression plasmids of Nodal and ALK7ca has been described. ${ }^{30}$ In another experiment, cells were treated with $500 \mathrm{ng} / \mathrm{ml}$ of $\mathrm{rNodal}$ (R\&D Systems). A wound was made using a $20-\mu \mathrm{L}$ pipette tip 24 hours after transfection or before incubation with rNodal. Ten points were marked randomly down the wound, and pictures were taken at the same points 24 hours after the wound was made. The distance migrated by the cells was measured using SimplePCl software (Compix Inc., Cranberry Township, PA).

\section{Transwell Migration and Invasion Assay}

Cell invasion was assessed by the ability of cells to digest and invade the Matrigel-coated $5 \mu \mathrm{m}$ pore size polycarbonate membrane Transwell inserts (Costar, Corning Inc., Corning, NY). Before cell seeding, inserts were coated with $100 \mu \mathrm{L}$ of growth-factor-reduced Matrigel (BD Biosciences), diluted 1:20. The Matrigel coating was allowed to solidify at room temperature under sterile conditions. Once dried, it was then reconstituted at $37^{\circ} \mathrm{C}$ for 1 hour with serum-free RPMI 1640. To determine the effect of overexpression and knockdown of Nodal and ALK7, cells were transfected with Nodal and ALK7-ca plasmid DNA or siRNAs for Nodal, ALK7, or ALK4 (see Table 2 for sequences) using Lipofectamine 2000. In some experiments, cells were transfected with Nodal or 
its empty vector as the control. The cells were treated with a proliferation inhibitor, mitomycin C (Sigma-Aldrich Corp.), $10 \mu \mathrm{g} / \mathrm{ml}$, for 2 hours. Transfected cells were then collected using Accutase (Innovative Cell Technologies Inc., San Diego, CA) and were seeded at a density of 20,000 cells per well on the Matrigel-coated filters. For rNodal treatment experiments, cells were incubated with RPMI 1640 medium supplemented with $1 \%$ fetal bovine serum and rNodal at different concentrations, whereas in the outer well of the Transwells, the medium was supplemented with $10 \%$ fetal bovine serum. At various points after plating, cells were fixed and stained with Harleco Hemacolor Staining Kit (EMD Chemicals, Gibbstown, $\mathrm{NJ}$ ), and noninvaded cells on the top of the filter were scraped off using a cotton swab. Filters were then separated from the wells using a scalpel and were mounted on slides for microscopic analysis. Invaded cells from 10 random fields at $\times 100$ magnification were counted under the microscope. Migration assays were performed using the same procedure except that the filters were not coated with Matrigel. The invasion/migration index was calculated as the fold of invaded/migrated cells in the treated groups over the control group.

\section{Protein Extraction and Immunoblot Analysis}

Cell and tissue lysates were prepared using radioimmunoprecipitation assay buffer $(50 \mathrm{mmol} / \mathrm{L}$ Tris- $\mathrm{HCl}, 150$ $\mathrm{mmol} / \mathrm{L} \mathrm{NaCl}, 1 \%$ Triton $\mathrm{X}-100,0.5 \%$ deoxycholate, and $1 \%$ SDS) containing $1 \mathrm{mmol} / \mathrm{L}$ dithiothreitol, $1 \mathrm{mmol} / \mathrm{L}$ $\mathrm{Na}_{3} \mathrm{VO}_{4}, 5 \mathrm{mmol} / \mathrm{L} \mathrm{NaF}, 100 \mathrm{mmol} / \mathrm{L}$ EDTA, $10 \mathrm{mg} / \mathrm{ml}$ aprotinin, and $100 \mu \mathrm{mol} / \mathrm{L}$ phenylmethylsulfonyl fluoride. Equal amounts of protein samples were separated by SDS-polyacrylamide gel electrophoresis and were transferred to a polyvinylidene difluoride membrane (Immobilon-P, Millipore Corp., Bedford, MA). After blocking with $5 \%$ milk in Tris-buffered saline Tween-20, the membrane was incubated with primary antibody overnight at $4^{\circ} \mathrm{C}$. The membranes were subsequently probed using horseradish-peroxidase-conjugated secondary antibody at room temperature for 1 hour. Signals were detected using the ECL Plus Kit (Amersham Biosciences, Piscat-
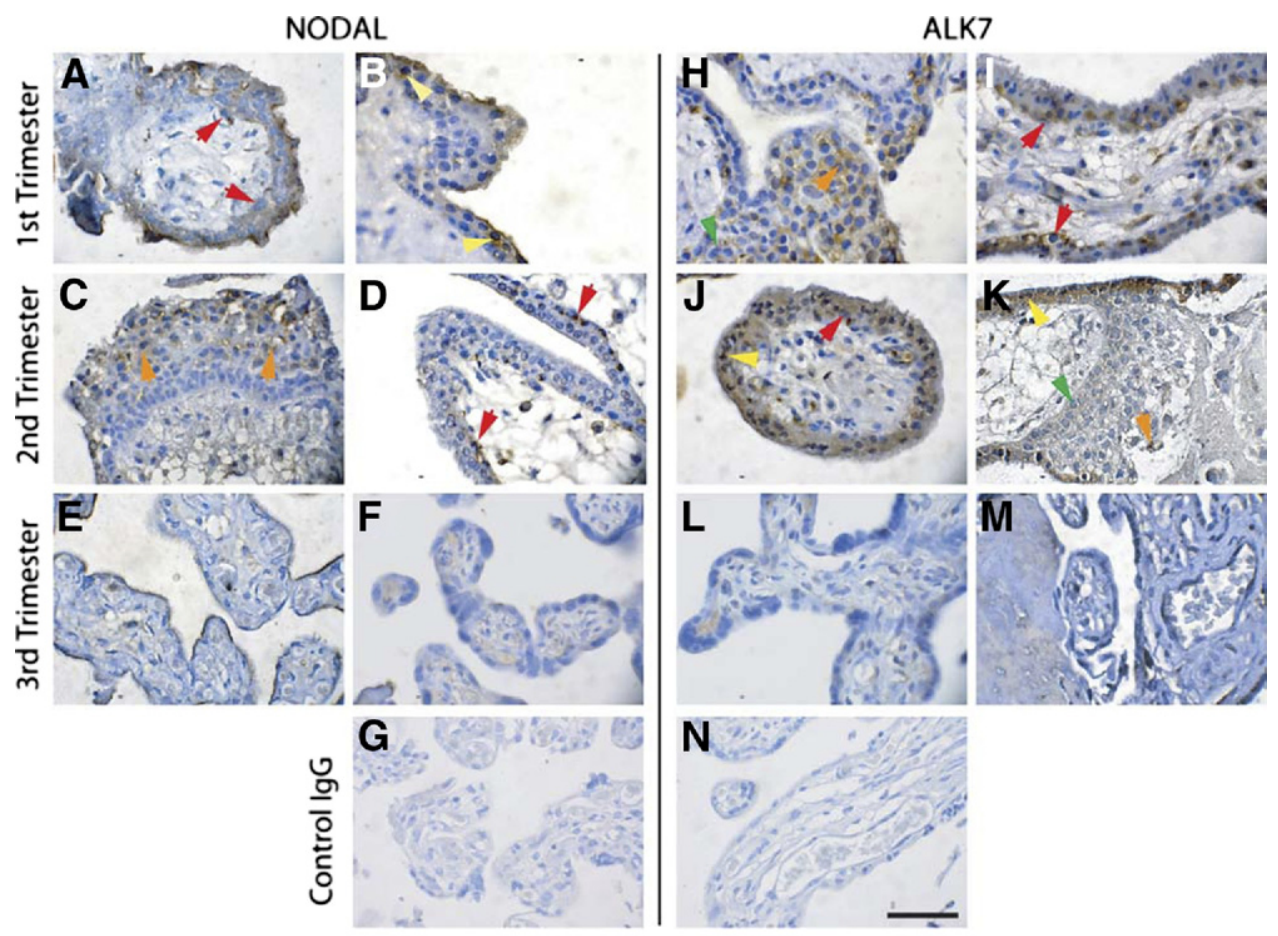

\begin{tabular}{|l|c|c|c|c|c|c|}
\multicolumn{2}{c}{} & \multicolumn{2}{c}{ 1st Trimester } & \multicolumn{2}{c}{ 2nd Trimester } & \multicolumn{2}{c|}{ 3rd Trimester } \\
\hline Gestational Age of Samples Analyzed & \multicolumn{2}{c|}{$5-12$ Week } & \multicolumn{2}{c|}{$13-19$ Week } & \multicolumn{2}{c|}{$30-40$ Week } \\
\hline Antigen Detected & Nodal & ALK7 & Nodal & ALK7 & Nodal & ALK7 \\
\hline Syncytial Trophoblasts (STB) & ++ & ++ & ++ & ++ & $+/-$ & $+/-$ \\
\hline Cytotrophoblasts (CTB) & +++ & +++ & ++ & ++ & $+/-$ & $+/-$ \\
\hline Column Cytotrophoblasts/proximal CC & ++ & +++ & $+/-$ & + & - & - \\
\hline Extravillous Cytotrophoblasts/Distal CC & +++ & +++ & ++ & + & - & - \\
\hline Mesenchyme & + & + & + & + & + & + \\
\hline Blood Vessels & $+/-$ & $+/-$ & + & + & + & ++ \\
\hline Syncytial Knots (SN) & ++++ & ++++ & ++++ & ++++ & ++++ & ++++ \\
\hline Number of Samples Analyzed & \multicolumn{2}{|c|}{13} & \multicolumn{2}{|c|}{9} \\
\hline
\end{tabular}

Figure 1. Expression of Nodal and ALK7 in placenta at different stages of pregnancy. First third-trimester placentae were subjected to IHC analyses using anti-Nodal (A-F), anti-ALK7 (H-M) antibodies, and goat (G) or rabbit (N) IgG control. Arrows represent positive staining for different cell types: CTB (red arrows), STB (yellow arrows), column CTBs (CC) (green arrows), and EVTs (orange arrows). Scale bar $=100 \mu \mathrm{m}$. The table depicts the scoring intensity of Nodal and ALK7 signals found in different cell types of blinded samples. - , no signal; $-/+$, no or weak signals; + , weak signal, ++ , moderate signal; +++ , strong signal; and ++++ , strongest signal. 
away, NJ). The following primary antibodies were used: anti-c-Myc (1:500; Cell Signaling Technology Inc., Beverly, MA), anti-phospho Smad2 (1:500; Cell Signaling Technology Inc.), anti-ALK7 (1:500; R\&D Systems), antiALK4 (1:500; BD Biosciences), and anti-Nodal (1:500; Santa Cruz Biotechnology). The membranes were reprobed with $\beta$-actin (1:10,000; Sigma-Aldrich Corp.) to confirm equal loading of protein samples.

\section{Tissue Inhibitor of Metalloproteinase-1 Immunoassay and Gelatin Zymography}

Tissue inhibitor of metalloproteinase-1 (TIMP-1) concentrations in the conditioned media from control plasmid, ALK7-ca, and Nodal-transfected cells or control and rNodal-treated explants were determined using a TIMP-1 enzyme-linked immunosorbent assay kit (Quantikine; R\&D Systems) following the manufacturer's instructions. Matrix metalloproteinase (MMP)-2/9 activity in the conditioned media or control and rNodal-treated HTR-8/SVneo cells was measured using gelatin zymography. Proteins were resolved on an $8 \%$ polyacrylamide gel containing $0.1 \%$ gelatin. The gel was then washed for 1 hour in $2.5 \%$ Triton X-100 solution (Roche Diagnostics GmbH, Mannheim, Germany) to renature the proteins and then washed with distilled water and incubated overnight with gentle shaking in $0.05 \mathrm{M}$ Tris- $\mathrm{HCl}$ with $5 \mathrm{mmol} / \mathrm{L} \mathrm{CaCl}_{2}$ for activation of MMPs. Gels were then soaked in $100 \mathrm{mmol} / \mathrm{L}$ EDTA for 15 minutes, fixed, and then stained with Coomassie Blue solution for 1 hour on a shaker. The gels were then soaked in destaining buffer until the desired resolution was obtained.

\section{RNA Extraction and RT-PCR}

Levels of TIMP-1 mRNA were determined using RT-PCR. HTR-8/SVneo cells were transfected with Nodal or its control vector overnight and were recovered for 2 to 48 hours. Total RNA was extracted using TRIzol reagent (Invitrogen), and RT-PCR was performed as described previously. ${ }^{30}$ Primers for glyceraldehyde-3-phosphate dehydrogenase have been previously published, ${ }^{10}$ and primers used to detect TIMP-1 were as follows: forward, 5'-AAGGCTCTGAAAAGGGCTTCC-3'; and reverse, 5'CAGGCTTCAGCTTCCACTCC-3'.

\section{Cytotoxicity Assay}

Conditioned media from HTR-8/SVneo cells transfected with control, Nodal, or ALK7-ca plasmid DNAs for 24 and 48 hours were collected and centrifuged. Cytotoxicity was measured using a CytoTox-ONE Assay Kit (Promega Corp., Madison, WI) which measures the release of lactate dehydrogenase (LDH) from nonviable cells. The $L D H$ substrate was added to the conditioned media (1:1 dilution), incubated at $22^{\circ} \mathrm{C}$ for 10 minutes in a 96-well lightprotected FluoroBlok plate (BD Biosciences), and fluorescence was measured at 560/590 nm. Cytotoxicity was determined using the following formula: \% Cytotoxicity = $100 \times$ (Experimental - Culture Medium Background)/ (Maximum LDH Release - Culture Medium Background).
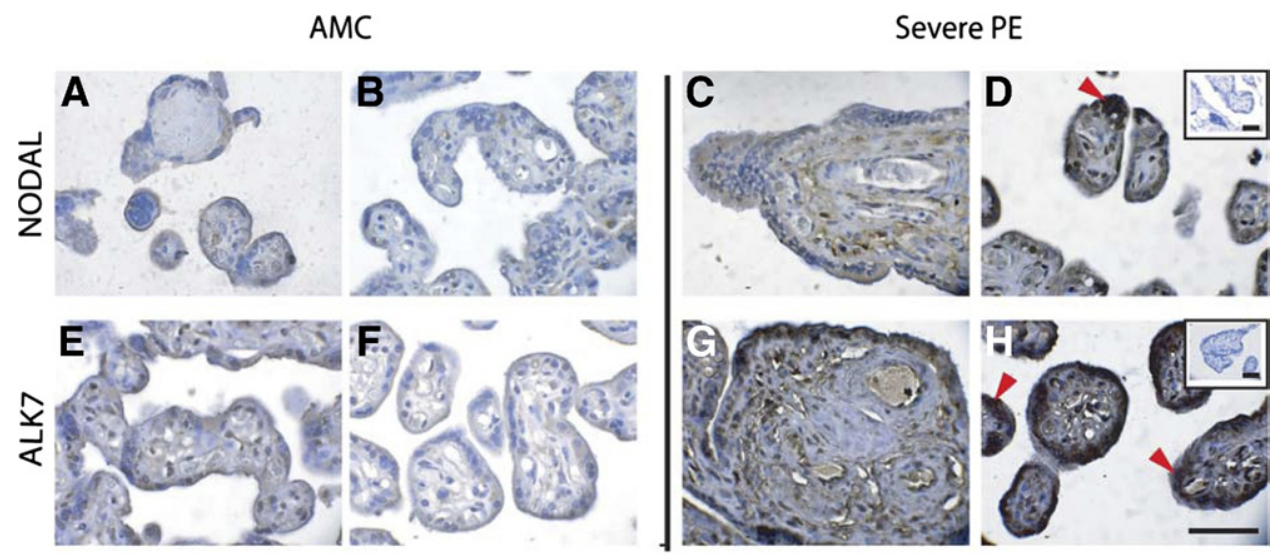

\begin{tabular}{|l|c|c|c|c|}
\hline & \multicolumn{2}{|c|}{ CONTROL } & \multicolumn{2}{c|}{ PE } \\
\hline Gestational Age of Samples Analyzed & \multicolumn{2}{|c|}{$28-34$ Week } & \multicolumn{2}{c|}{$27-33$ Week } \\
\hline Antigen Detected & Nodal & ALK7 & Nodal & ALK7 \\
\hline Syncytial Trophoblasts (STB) & - & + & ++ & +++ \\
\hline Cytotrophoblasts (CTB) & $+/-$ & + & + & +++ \\
\hline Mesenchyme & + & + & ++ & +++ \\
\hline Blood Vessels & $+/-$ & + & $+/-$ & ++ \\
\hline Syncytial Knots (SN) & ++++ & ++++ & ++++ & ++++ \\
\hline Number of Samples Analyzed & \multicolumn{2}{|c|}{8} \\
\hline
\end{tabular}

Figure 2. Aberrant expression of Nodal and ALK7 in preeclamptic placenta. Immunohistochemical staining of Nodal (A-D) and ALK7 (E-H) in placentae from patients with severe preeclampsia (PE) or gestational age-matched controls (AMCs). Insets depict IgG controls. Red arrows indicate the SN. Scale bars: $100 \mu \mathrm{m}$. The table summarizes the scoring intensity of positive staining found in different cell types of blinded samples. - , no signal; $-/+$, no or weak signals; + , weak signal, ++ , moderate signal; +++ , strong signal; and ++++ , strongest signal. 
A

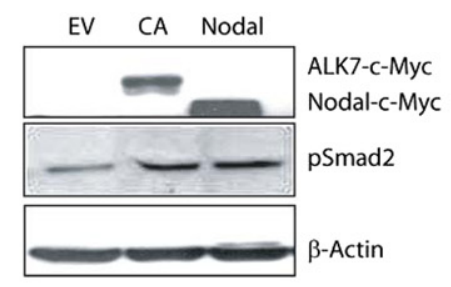

B
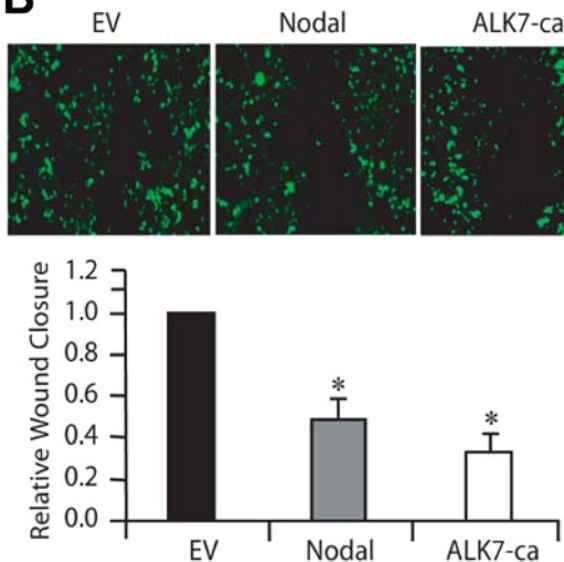

C
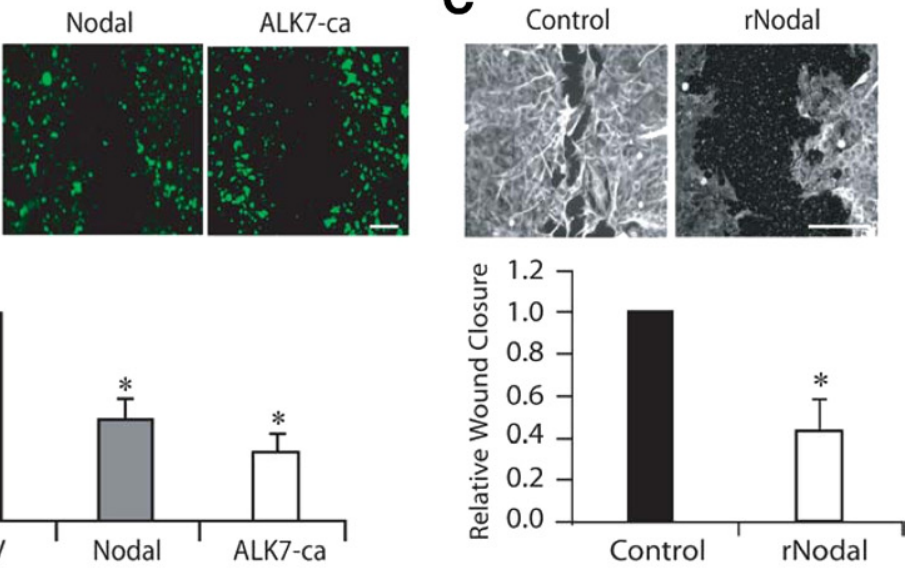

D

EV

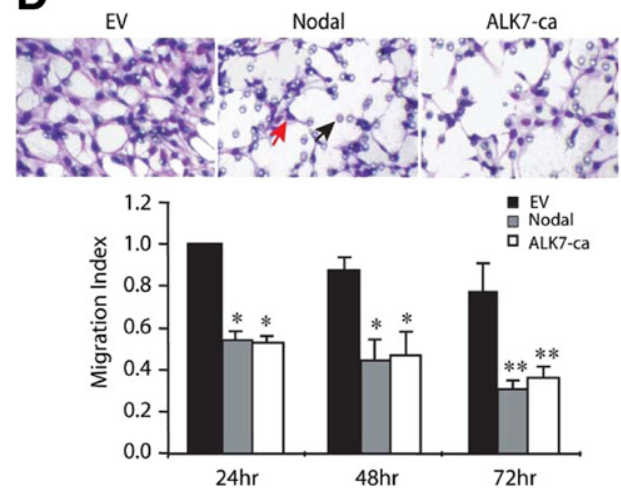

Figure 3. Overexpression of Nodal and ALK7-ca inhibited trophoblast migration. A: Confirmation of Nodal and ALK7-ca expression and their activation of Smad2 signaling in HTR-8/SVneo cells transfected with control empty vector plasmid (EV), Nodal, or ALK7-ca (CA). B: Wound-healing assay. HTR-8/SVneo cells were transiently transfected with Nodal, ALK7-ca, or EV, and a wound was created. Migration of cells into the wounded area was monitored and measured. Scale bar $=100$ $\mu \mathrm{m} .{ }^{*} P<0.05$ versus EV $(n=3)$. C: Wound-healing assay performed with treatment of rNodal (500 $\mathrm{ng} / \mathrm{ml})$ and its vehicle control. ${ }^{*} P<0.05$ versus control $(n=5)$. Scale bar $=100 \mu \mathrm{m}$. D: Transwell migration assay performed with HTR-8/SVneo cells transiently transfected with Nodal, ALK7-ca, or EV control and plated on the top of the Transwells. Cells migrated (red arrow) through the pores (black arrow) were counted 24 to 72 hours after plating. ${ }^{*} P<0.05$ and ${ }^{* * *} P<0.01$ versus EV at the same time point $(n=4)$. Error bars represent SEM.

Maximum LDH release was determined by measuring $\mathrm{LDH}$ levels in the lysates of nontransfected cells or control explants.

\section{Statistical Analysis}

The data presented in all the figures are mean \pm SEM of independent experiments ( $n=3$ to 5). In most cases, data from different experiments were normalized to the control value before being combined for statistical analyses. Differences among several groups were determined using one-way ANOVA followed by the Student-Newman-Keuls test using GraphPad InStat software (GraphPad Software Inc., San Diego, CA). For comparison between two groups, the $t$-test was used. A $P<0.05$ was considered significant.

\section{Results}

\section{Spatial and Temporal Expression of Nodal and ALK7 in Normal and Preeclamptic Placenta}

Using IHC analysis, we first determined Nodal and ALK7 expression in placenta. During early pregnancy, Nodal and ALK7 immunoreactivities were detected in villous CTBs and STBs and in EVT cells (Figure 1, A, B, H, and I). Scoring of $\mathrm{IHC}$ signal intensity revealed that moderate signals of Nodal and ALK7 were present in the STBs of the first- and second-trimester placentae. Strong, and in some cases, moderate, Nodal and ALK7 immunoreactivities were observed in the villous CTBs and EVT cells in the first-trimester placentae. During the second trimester of pregnancy, however, Nodal and ALK7 signal intensity was down to weak to moderate levels. In anchoring villi of second-trimester placentae (13 to 19 weeks), expression of Nodal was mostly absent from the cells at the base of the cell column, the proliferative population of EVTs, although weak staining was detected in the more distal EVT population (Figure 1C). Weak ALK7 signals were observed in the proximal portion of the cell column and in some distal EVTs (Figure 1, H and K). In third-trimester placentae, Nodal and ALK7 immunoreactivities were dramatically decreased, with only weak signals detected mostly in the villous mesenchyme (Figure 1, E, F, L, and M). In all stages of pregnancy, the strongest expression signal for Nodal and ALK7 was found in the syncytial knots (SNs, the aggregates of apoptotic nuclei of STB) (Figure 1). A comparative analysis was made between the severe early-onset preeclamptic placental tissue and their gestational age-matched controls for the expression levels of Nodal and ALK7. Similar to the gestational expression profiles presented in Figure 1, Nodal and ALK7 levels were weak to nondetectable in controls. In contrast, much higher levels of Nodal and ALK7 expression were observed in the preeclamptic placenta (Figure 2). ALK7 was strongly expressed in the STB layer, CTBs, SNs, and villous mesenchyme, and moderate signals were also found around blood vessels. Nodal was found in the STBs and villous 
mesenchyme (Figure 2, A-H). Again, SNs exhibited the strongest signals for Nodal and ALK7 (Figure 2).

\section{Nodal Inhibits Trophoblast Migration and Invasion}

The first-trimester human trophoblast cell line HTR-8/ SVneo has been extensively used to study trophoblast cell migration and invasion. ${ }^{26,31}$ To determine the function of Nodal in trophoblast cell migration, we first performed wound-healing assays in HTR-8/SVneo cells. Cells were transiently transfected with Nodal, ALK7-ca, or the empty vector as a negative control. Overexpression of Nodal and ALK7-ca was assessed by means of Western blotting using an anti-c-Myc antibody (Figure 3A), and activation of Nodal signaling was confirmed by Smad2 phosphorylation levels (Figure 3A). To monitor the migration of transfected cells, cells were cotransfected with plasmid encoding enhanced green fluorescent protein plasmid DNA. A wound in the confluent cell layer was made 24 hours after transfection, and the ability of cells to migrate into the wounded area was monitored and quantified. Significant inhibition of the migration of cells to the wounded area was observed in cells transfected with Nodal or ALK7-ca (Figure 3B). Similarly, treatment with rNodal also inhibited the migration of cells into the wounded area (Figure 3C). Transwell migration assays were also performed to confirm the effect of Nodal and ALK 7 on cell migration. Cells were transfected with empty vector control, Nodal, or ALK7-ca. Twenty-four to 72 hours after transfection, the number of cells that migrated through the pores of the filter was significantly lower in Nodal- or ALK7-ca-transfected cells compared with empty vector-transfected cells (Figure 3D).

Similar experiments were performed using Matrigelcoated Transwell filters to assess cell invasion. Transfection of cells with Nodal or ALK7-ca led to a significant decrease in the number of cells invaded through the Matrigel, and this effect was observed 24 to 72 hours after transfection (Figure 4A). We previously observed that ALK7 and Nodal inhibit proliferation and induce apoptosis in trophoblast cells. ${ }^{11}$ To rule out the possibility that the decrease in the number of invaded and migrated cells in Nodal and ALK7-ca-transfected groups is entirely due to the decrease in cell proliferation, cells were transfected with Nodal plasmid DNA and then were treated with mitomycin $\mathrm{C}$ for 2 hours before plating on the Matrigel-coated filter. Treatment with mitomycin $\mathrm{C}$ decreased the number of cells invaded; however, it did not reverse the effect of Nodal. Overexpression of Nodal inhibited cell invasion in the absence or presence of mitomycin $\mathrm{C}$ (Figure 4B). Similar results were obtained in Transwell migration experiments (data not shown). Finally, the effect of Nodal on cell invasion was confirmed using rNodal treatment. Incubation with rNodal caused a dose-dependent inhibition of cell invasion, and this effect of Nodal was found to be significant at a dose of $100 \mathrm{ng} / \mathrm{ml}$ or higher (Figure 4C).

Because Nodal has been suggested to act through both ALK4 and ALK7, we determined which of these
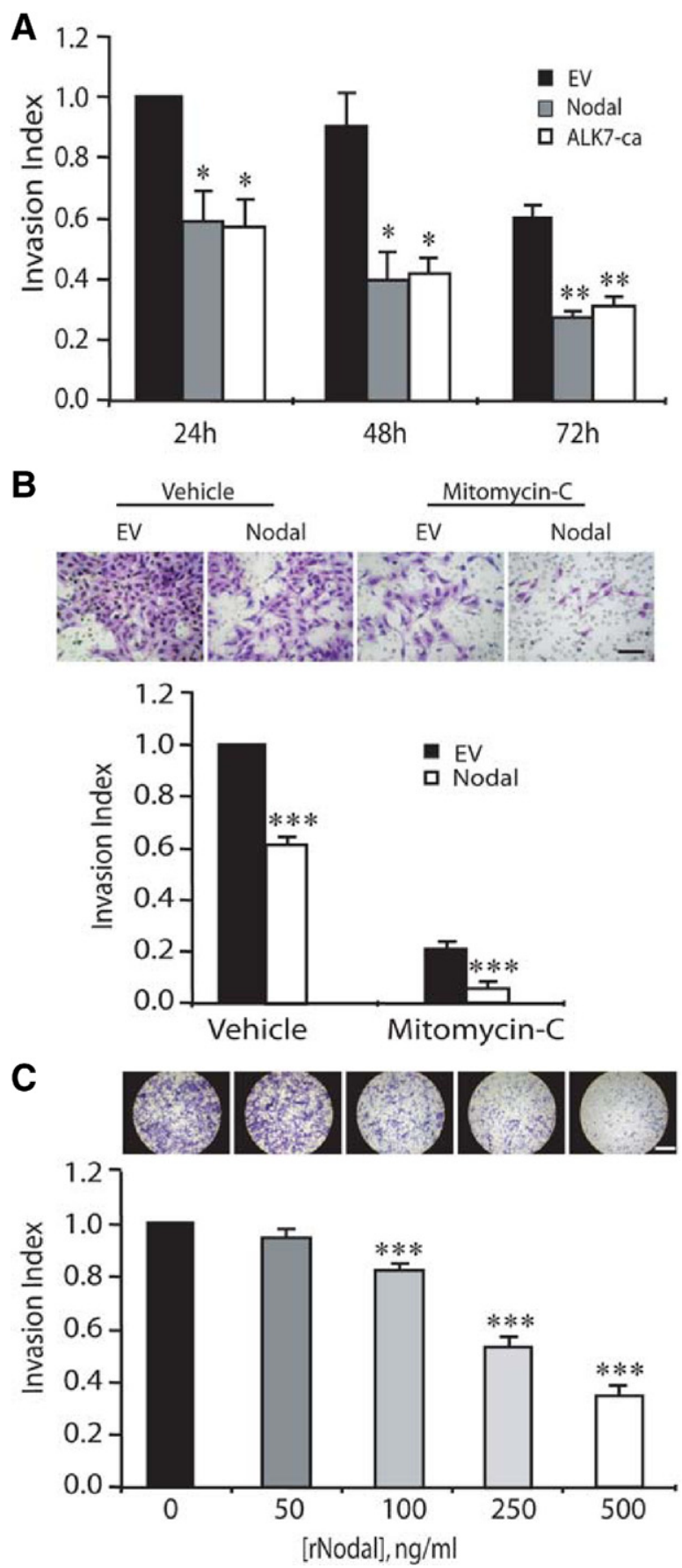

Figure 4. Nodal inhibited trophoblast cell invasion as determined by Transwell assays on Matrigel-coated filters with HTR-8/SVneo cells. A: Cells were transiently transfected with Nodal, ALK7-ca, or empty vector control (EV) and plated on the top of the Transwells. Twenty-four, 48, and 72 hours after plating, cells invaded through the pores were counted. ${ }^{*} P<0.05$ and ${ }^{* *} P<$ 0.01 versus $E V$ at the same time point $(n=4)$. B: Cells transiently transfected with Nodal or EV were treated with mitomycin C $(10 \mu \mathrm{g} / \mathrm{ml})$ or its vehicle control for 2 hours. Invaded cells were scored 24 hours after plating. Scale bar $=100 \mu \mathrm{m} .{ }^{* * *} P<0.001$ versus respective EV control $(n=3)$. C: Cells were treated with different concentrations of rNodal for 24 hours, and invaded cells were counted. Scale bar $=1 \mathrm{~mm}$. ${ }^{* * * *} P<0.001$ versus control $(n=3)$. Error bars represent SEM.

receptors mediates the effect of Nodal on cell invasion by using an siRNA-mediated gene silencing approach. Cells were transfected with control, ALK4, ALK7, or Nodal siRNAs, together with control vector or Nodal 
A

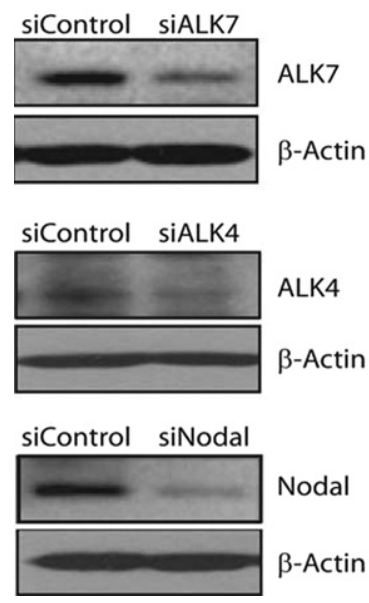

B

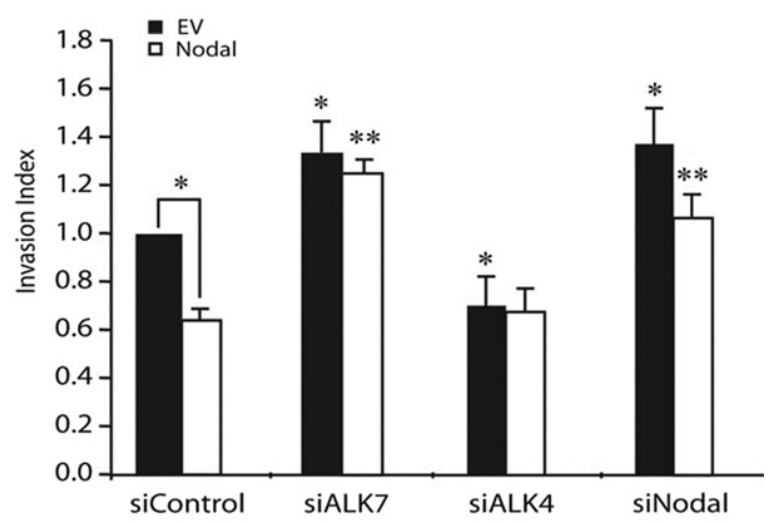

Figure 5. Effects of Nodal, ALK7, and ALK4 siRNAs on trophoblast invasion. A: Confirmation of knockdown efficiency of siRNAs. HTR-8/ SVneo cells were transiently transfected with 100 nmol/L control siRNA (siControl), ALK7 siRNA (siALK7), ALK4 siRNA (siALK4), or Nodal siRNA (siNodal). An equal amount of protein was analyzed by means of immunoblot analyses. B HTR-8/SVneo cells were transiently transfected with $100 \mathrm{nmol} / \mathrm{L}$ siControl, siALK7, siALK4, or siNodal, along with a control vector or Nodal plasmid. Invaded cells were quantified 24 hours after plating. Both siALK7 and siNodal reversed the effect of Nodal. ${ }^{*} P<0.05$ versus the group transfected with empty vector (EV) and siControl. ${ }^{* * *} P<0.05$ versus the group transfected with Nodal plasmid and siControl $(n=3)$. Error bars represent SEM. plasmid DNA. Nodal, ALK7, and ALK4 siRNAs reduced the expression of respective targets (Figure 5A). ALK7 and Nodal siRNAs significantly increased cell invasion, whereas ALK4 siRNA had the opposite effect (Figure 5B). Overexpression of Nodal decreased cell invasion in control siRNA-transfected cells, but this effect was completely reversed by ALK7 SiRNA and reduced by Nodal siRNA (Figure 5B). ALK7 siRNA also blocked rNodalinhibited cell invasion (data not shown).

\section{Nodal Inhibits Placental Explant Outgrowth}

Villous explant culture is an excellent model for studying EVT invasion because it not only preserves the actual orga- nization of the villous structure but also very closely mimics the in vivo physiologic microenvironment. ${ }^{32}$ Therefore, to further confirm the physiologic significance of Nodal in placental development, we tested the effect of rNodal and Nodal silencing on EVT outgrowths. Incubation with rNodal inhibited expansion of the EVT outgrowth across and into the Matrigel (Figure 6A), and the area of outgrowth was significantly lower in the rNodal-treated group than in the control group (Figure 6B). We performed $\mathrm{IHC}$ analysis of HLA-G1, a marker of invasive EVT, ${ }^{33}$ on control and rNodaltreated explants. Compared with control explants, rNodaltreated explants had fewer HLA-G-positive EVT cells and limited migration of these EVT cells into the Matrigel (Figure $6 \mathrm{C})$. In contrast, silencing of Nodal stimulated the expan-
A
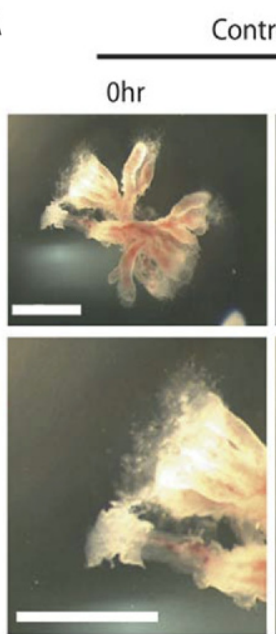

B

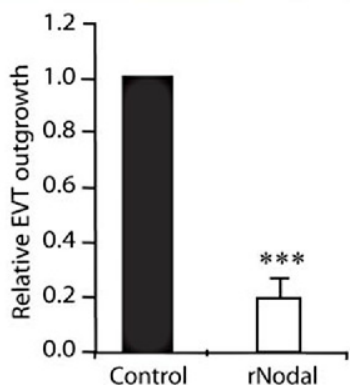

$72 \mathrm{hr}$
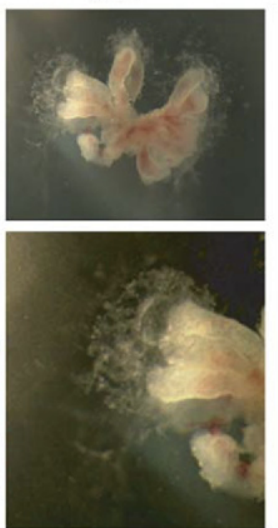

C

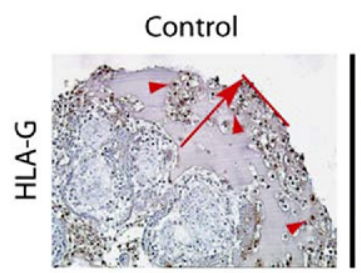

Nodal

Ohr
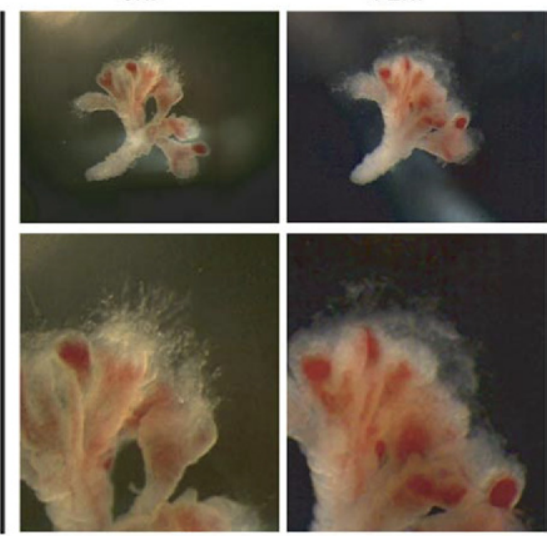

Figure 6. Nodal inhibited the outgrowth of firsttrimester placental explants. A: Representative pictures of explants from a 9-week placenta. The same explants were photographed before and 72 hours after treatment. Scale bars: $500 \mu \mathrm{m}$. B: Explants from 6- to 9-week placentae were cultured in the presence and absence of rNodal $(250 \mathrm{ng} / \mathrm{ml})$. The graph shows the relative EVT outgrowth 72 hours after rNodal treatment. ${ }^{\text {*a* }} P<0.001$ versus control $(n=4)$. C: IHC analysis of explants. Control and rNodal-treated explants were fixed, sectioned, and stained for HLA-G1, a marker of invasive EVT cells. Red arrowheads indicate HLA-G1-positive cells, and red arrows indicate the distance traveled by the EVTs on the Matrigel. Treatment with rNodal resulted in retarded invasion compared with control. Inset: Mouse IgG control. Scale bars: $200 \mu \mathrm{m}$. Error bars represent SEM. 
A
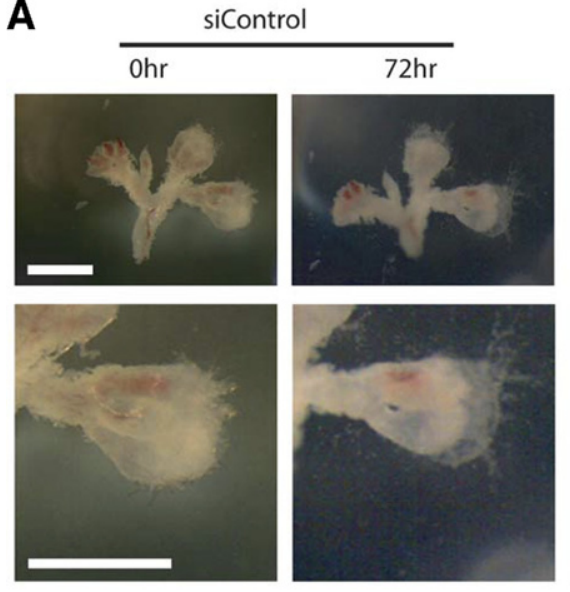
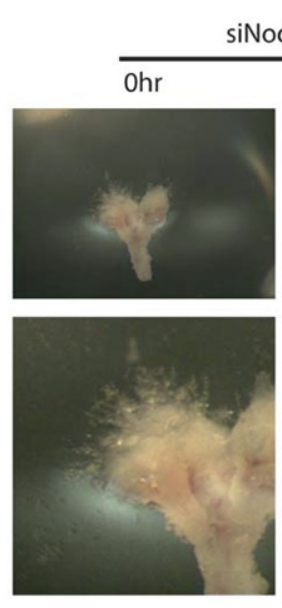

D

C

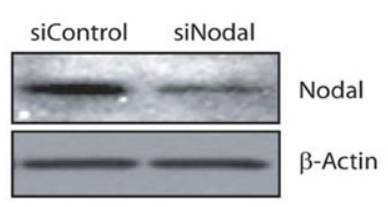

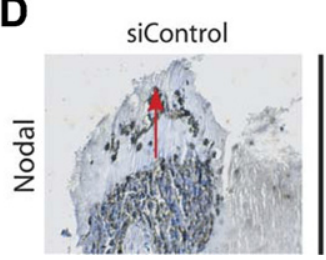

siNodal

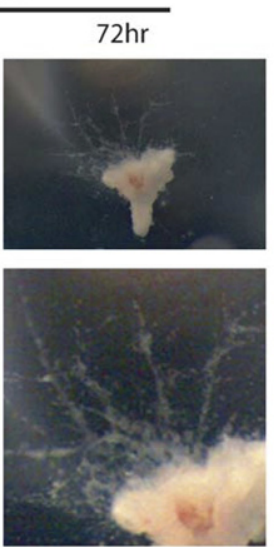

B

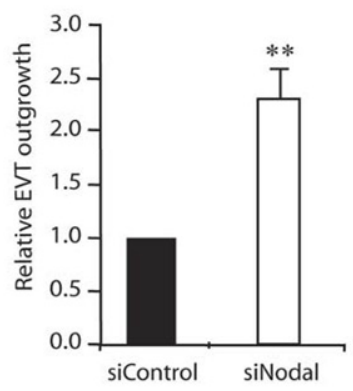

Figure 7. Nodal siRNA (siNodal) strongly promoted the outgrowth of first-trimester placental explants. A: Representative pictures of explants from a 9-week placenta. The same explants were photographed before and 72 hours after treatment. Scale bars: $500 \mu \mathrm{m}$. B: Graph showing relative EVT outgrowth 72 hours after siRNA treatment. ${ }^{* *} P<0.01$ versus control $(n=4)$. C: Confirmation of the knockdown effect of siNodal in explants. Explants were cultured with 100 $\mathrm{nmol} / \mathrm{L}$ siNodal or control siRNA (siControl). Protein was extracted 48 hours after siRNA treatment and was analyzed by means of Western blotting. D: Nodal immunoreactivity in siControl- and siNodal-transfected explants. Red arrows indicate the distance traveled by the EVTs on the Matrigel Scale bar $=200 \mu \mathrm{m}$. Error bars represent SEM.

sion of explants and increased the number of streams of EVTs that extended across the Matrigel surface (Figure 7, A and D). Quantitative analysis revealed that knockdown of Nodal caused a significant increase in the area of outgrowth (Figure 7B). Nodal siRNA decreased the expression of Nodal, as confirmed by immunoblotting (Figure $7 \mathrm{C}$ ) and $\mathrm{IHC}$ analysis (Figure 7D).

\section{The TIMP-1-MMP-2/MMP-9 Pathway Is Involved in Nodal/ALK7-Regulated Cell Migration and Cell Invasion}

The invasion of EVT cells depends on the degradation of extracellular matrix by MMP-2 and MMP-9, and their activity is, in turn, controlled by TIMP-1. ${ }^{34}$ We hypothesized that Nodal inhibits trophoblast cell invasion by increasing TIMP-1 secretion and decreasing MMP-2 and MMP-9 activity. To test this possibility, we first used enzymelinked immunosorbent assay to measure TIMP-1 concentrations in the conditioned media of control-, Nodal-, or ALK7-ca-transfected cells. In a time course experiment, we found that Nodal and ALK7-ca increased TIMP-1 secretion 24 and 48 hours after transfection (Figure 8A). To rule out the possibility that the increase in TIMP-1 levels in the conditioned media is entirely due to the release of the enzyme from dead cells, we measured LDH levels as an indicator of cytotoxicity. There was a small increase in LDH levels in the conditioned media of Nodal- and ALK7-ca-transfected cells at 24 and 48 hours (Figure 8A, right). In placental explant culture, treatment with rNodal also induced a strong increase in TIMP-1 secretion (Figure 8B). To further confirm the regulation of TIMP-1 by Nodal, cells were transfected with Nodal or its control vector, and at various time points after transfection, RNA was extracted and TIMP-1 mRNA levels were determined by means of RT-PCR. An increase in TIMP-1 mRNA levels was observed in the Nodal-transfected group compared with controls 6, 12, and 24 hours after transfection (Figure $8 \mathrm{C})$. Nodal also inhibited MMP-2/9 activity as determined by gelatin zymography (Figure 8D). To confirm that TIMP-1 is involved in Nodal/ALK7-regulated cell invasion, we tested the effect of Nodal and ALK7-ca in the presence of a TIMP-1-neutralizing antibody. As expected, overexpression of Nodal and ALK7-ca decreased the invasion index. In the presence of control IgG, the effect of ALK7-ca and Nodal was slightly enhanced. However, the anti-TIMP-1 antibody significantly reversed the effect of Nodal and ALK7-ca on cell invasion (Figure 8E).

\section{Discussion}

Previously, we reported that Nodal acts through ALK7 to regulate trophoblast cell proliferation and apoptosis. ${ }^{11}$ In this study, we further examined the role of Nodal/ALK7 signaling in human placenta. We found that Nodal and ALK7 are expressed in human placenta and are upregulated in preeclampsia. We demonstrated that the Nodal/ALK7 pathway is involved in regulating migration and invasion of trophoblast cells and that the TIMP-1MMP-2/MMP-9 pathway partially mediates the effect of Nodal/ALK7 on trophoblast cell migration/invasion.

Using $\mathrm{IHC}$ analysis, we demonstrated that Nodal and ALK7 are expressed in human placenta and that their expression is developmentally regulated. Consistent with their functions in regulating trophoblast apoptosis, ${ }^{11}$ Nodal and ALK7 are highly expressed in SNs, which consist of aggregates of apoptotic nuclei. ${ }^{35}$ Nodal and 
A

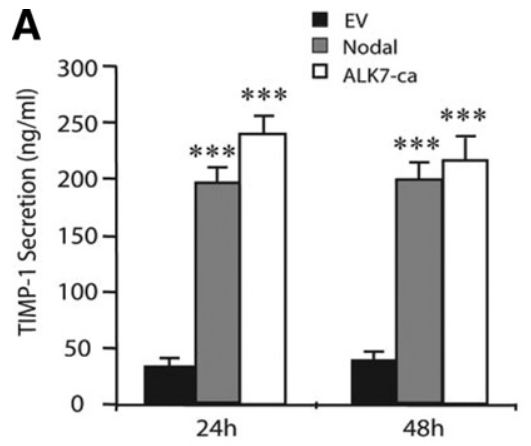

C

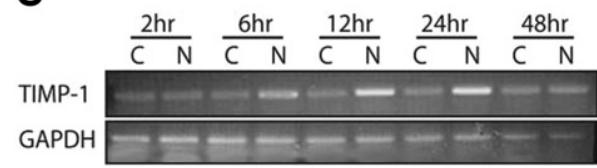

D

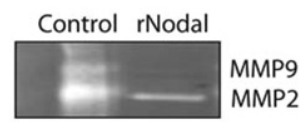

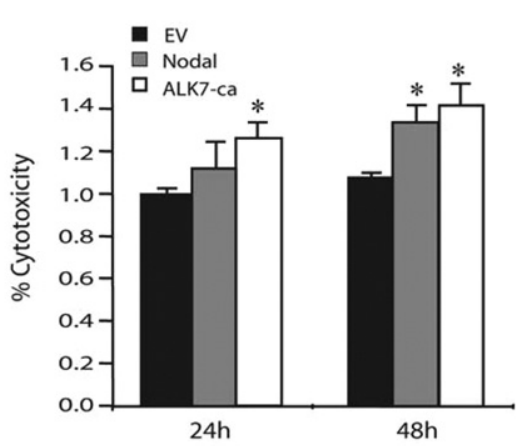

B

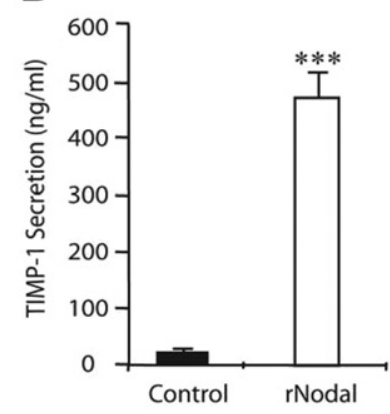

E

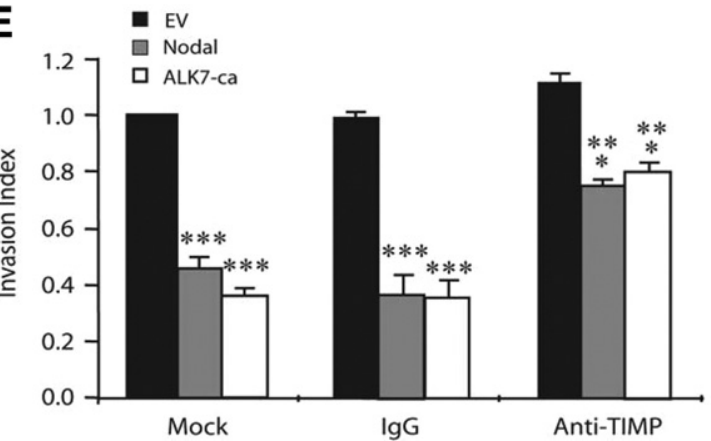

Figure 8. The TIMP-1 partially mediates the anti-invasive effect of Nodal. A: Nodal and ALK7-ca induced TIMP-1 secretion. HTR-8/SVneo cells were transiently transfected with Nodal, ALK7-ca, or their empty vector control (EV). Twenty-four and 48 hours after transfection, conditioned media were collected and subjected to TIMP-1 (left) and LDH (right) measurement. ${ }^{*} P<0.05$ and ${ }^{* * * * *} P<0.001$ versus EV at the same time point $(n=3)$. B: Nodal induced TIMP-1 secretion in villous explant culture. Explants were treated with rNodal $(500 \mathrm{ng} / \mathrm{ml})$, and TIMP-1 secretion into the media was measured 48 hours after treatment. ${ }^{* * * *} P<0.001$ versus control $(n=3)$. C: Nodal increased TIMP-1 mRNA levels in a time-dependent manner. HTR-8/SVneo cells were transfected with EV $(\mathrm{C})$ or Nodal $(\mathrm{N})$. Total RNA was extracted at the indicated time points, and RT-PCR was performed using TIMP-1 primers. GAPDH, glyceraldehyde-3phosphate dehydrogenase, was used as internal control. D: Nodal inhibited MMP-2 and MMP-9 activity. Cells were treated with rNodal for 24 hours, and conditioned media were subjected to gelatin zymography. E: Anti-TIMP-1 antibody partially reversed the anti-invasive effect of Nodal and ALK7-ca. Cells were transfected with Nodal, ALK7-ca, or their EV and plated on the top of Transwells in the presence of culture medium only (Mock), control IgG, or anti-TIMP-1 antibody. ${ }^{*} P<0.05$ and ${ }^{* * * *} P<0.001$ versus EV; and ${ }^{* *} P<0.05$ versus Nodal or ALK7-ca of Mock and IgG control $(n=3)$. Error bars represent SEM

ALK7 can be seen in CTBs and STBs, supporting their function in regulating trophoblast proliferation and suggesting that they may also play a role in STB differentiation. The possibility that Nodal may regulate STB differentiation is supported by mouse model data wherein a hypomorphic mutation of Nodal resulted in deceased labyrinth formation and an increased number of giant and spongiotrophoblast cells. ${ }^{8}$ In support of the finding that Nodal and ALK7 regulate cell invasion, both molecules are expressed in distal and proximal EVTs in early pregnancy. However, starting at week 13 of gestation, Nodal and ALK7 levels in cells at the base of anchoring cell columns and in EVTs at the distal areas become very weak or nondetectable, suggesting that a decrease in their signaling strength at this time is important to allow more trophoblast proliferation and invasion into the uterus. Factors responsible for such dynamic regulation of Nodal and ALK7 expression remain to be identified; however, a potential regulator is oxygen tension. A role for oxygen tension in human placental development has been established, ${ }^{13,36,37}$ and the related factor transforming growth factor- $\beta 3$, which inhibits trophoblast differentiation into the invasive pathway, is up-regulated by hypoxia-inducible factor-1. ${ }^{13,38}$ The possibility that hypoxia regulates Nodal and ALK7 expression is currently under investigation.

Trophoblast cell invasion into the uterus is an essential process for successful pregnancy. This process takes place within the first 20 weeks of pregnancy ${ }^{39}$ and is tightly regulated by a network of positive and negative regulatory factors whose expression are dynamically regulated during gestation. ${ }^{12,14,40}$ In this study, we provide evidence that Nodal is a physiologic regulator of trophoblast invasion. Using $\mathrm{IHC}$ analysis, we showed that Nodal and its receptor, ALK7, are expressed in invasive EVT cells during early pregnancy. In an EVT cell model, HTR8/SVneo, we found that overexpression of Nodal or incubation with rNodal inhibited, whereas silencing of Nodal expression increased, trophoblast cell migration and invasion, as revealed by wound-healing, Transwell migration, and Transwell invasion assays. Consistent with findings in HTR-8/SVneo cells, we observed that Nodal treatment suppressed outgrowth of placental explants and that Nodal siRNA stimulated the expansion and invasion of EVT outgrowths. Because we found that Nodal inhibits trophoblast cell proliferation and induces apoptosis, we cannot exclude the possibility that these effects also contribute to the decrease in the number of cells that have migrated/invaded through the Transwell and the inhibition of explant outgrowth. However, these results clearly support a direct role of Nodal in regulating trophoblast cell migration and invasion. We observed that in the presence of mitomycin C (proliferation inhibitor), Nodal still inhibited cell invasion. In addition, in explants treated with Nodal there was a clear decrease in the number and spread of HLA-G1-positive cells, whereas in Nodal siR- 
NA-transfected explants, the outgrowth of EVT cells was strongly enhanced. These results suggest that Nodal may inhibit the differentiation of invasive EVTs. Finally, Nodal up-regulated TIMP-1 expression and secretion and down-regulated MMP-2/9 activities. Higher TIMP-1 secretion and lower MMP-2/9 activities have been linked to inhibition of trophoblast cell invasion. ${ }^{14}$

Although Nodal has been shown to signal through two type I receptors, ALK4 and ALK7, several findings from this study suggest that the inhibitory effect of Nodal on trophoblast cell migration and invasion is mainly mediated by ALK7. First, constitutively active ALK7 had a similar inhibitory effect as Nodal on trophoblast cell migration/invasion. Second, knockdown of ALK7 and Nodal using siRNAs had similar effects on enhancing cell invasion, whereas knockdown of ALK4 had opposite effects. It has been reported that activin-A, which also signals through ALK4, stimulates trophoblast invasion and explant outgrowth. ${ }^{41}$ Therefore, the decrease in cell invasion after ALK 4 knockdown is likely due to the inhibition of activin signaling. Taken together, these data strongly support the role of ALK7 in Nodal-inhibited cell migration/ invasion.

In this study, we found that the inhibitory effect of the Nodal/ALK7 pathway on cell invasion/migration is mediated, at least in part, through the TIMP-1-MMP-2/ MMP-9 pathway. Overexpression of ALK7-ca or Nodal significantly increased TIMP-1 secretion and correspondingly decreased the activity of MMP-2 and MMP-9. The TIMP-1 neutralizing antibody significantly, but not completely, reversed the effect of Nodal on trophoblast cell invasion. The MMPs are implicated in normal cell invasion and migration by degrading the extracellular matrix. High levels of MMP-9 are expressed by CTB cells, and blocking of MMP-9 with an antibody can abrogate their invasion of the extracellular matrix in vitro. ${ }^{42}$ Neutralization of TIMP-1 activity did not completely block the effect of Nodal, suggesting that Nodal may act through additional pathways to exert the anti-invasive effect.
Preeclampsia is a serious pregnancy complication. Although the causes of preeclampsia are not entirely clear, shallow trophoblast invasion and excessive apoptosis have been suggested to play a role in its development. ${ }^{16,18,43}$ In this study, we provided the first evidence, to our knowledge, that abnormal signaling of Nodal may play a role in the development of preeclampsia. We found that Nodal and ALK7 expression are strongly up-regulated in trophoblasts of preeclamptic placenta. In severe early-onset preeclamptic placenta, we also detected Nodal and ALK7 signals in the villous mesenchyme surrounding the paravascular capillary network of the intermediate villi. This novel observation suggests that Nodal may also have a role in either angiogenesis or vascular control. We postulate that the failure in downregulation of Nodal and ALK7 levels may be associated with, or contribute to, the development of preeclampsia. If Nodal/ALK7 signaling strength in EVTs remains high during early second-trimester pregnancy, it may result in limited CTB proliferation and shallow invasion of EVTs into the uterus. Consistent elevation of Nodal/ALK7 signaling would also contribute to the excessive apoptosis and shedding of the STBs that is known to occur in preeclampsia. ${ }^{43}$ Furthermore, oversignaling of the Nodal/ALK7 pathway could lead to an increase in TIMP-1 secretion and a decrease in MMP-2 activity. An imbalance in the MMP-2/TIMP-1 ratio has been found in women who develop preeclampsia. ${ }^{44}$ Thus, up-regulation of Nodal and ALK7 may contribute to the pathogenesis of preeclampsia.

Based on these novel findings that i) Nodal and ALK7 expression in normal placenta is spatially and developmentally regulated, ii) Nodal and ALK7 are aberrantly expressed in preeclamptic placenta, and iii) Nodal acts through ALK7 to inhibit trophoblast cell proliferation, migration, and invasion and to induce trophoblast apoptosis, we propose that Nodal and ALK7 play important roles in maintaining normal placental development and that their abnormal signaling may contribute to the development of preeclampsia (Figure 9A). Since the role of
A

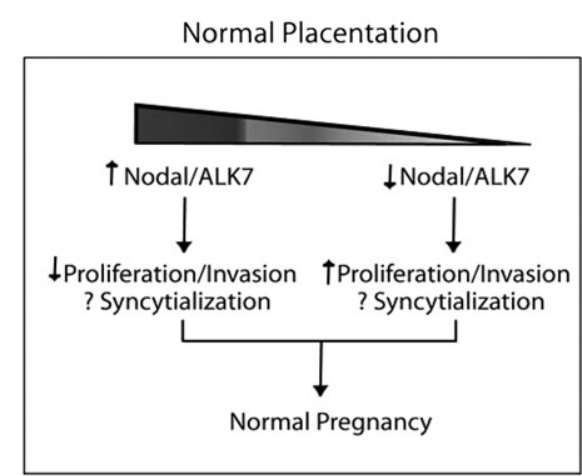

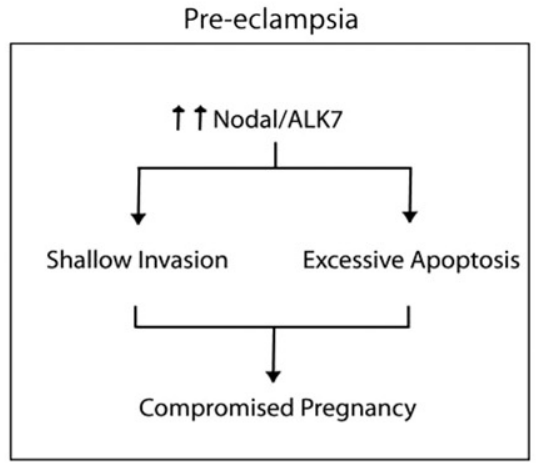

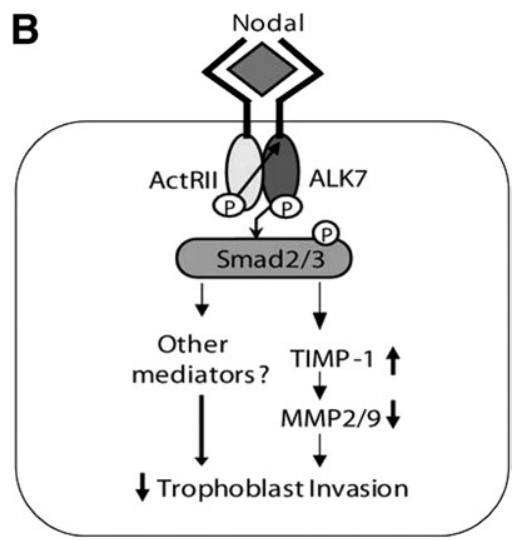

Figure 9. Proposed role of Nodal in human placental development and mechanism of Nodal-regulated trophoblast invasion. A: In normal placenta, high expression of Nodal/ALK7 during the first trimester of pregnancy inhibits trophoblast proliferation and invasion and may also play a role in syncytialization. The subsequent decrease in Nodal/ALK7 levels will allow higher proliferation and invasion of trophoblast cells. The pathway may interact with other stimulatory and inhibitory signaling pathways to ensure normal placentation. In preeclamptic placenta, Nodal and ALK7 are overexpressed. The aberrant up-regulation of this pathway may result in limited trophoblast invasion and excessive apoptosis, thereby contributing to the development of preeclampsia. B: Nodal acts through the ALK7 and Smad2/3 pathway to induced TIMP-1 secretion, which, in turn, inhibits MMP-2/9 activity, leading to a decrease in cell invasion. Other pathways involved remain to be identified. ActRII, activin receptor II. 
TIMP-1 and MMP-2/MMP-9 in trophoblast invasion is well established and Nodal modulates TIMP-1 secretion and MMP-2/9 activity, we also proposed that TIMP-1 and MMP-2/MMP-9 are involved in Nodal/ALK7-induced inhibition of invasion and migration (Figure 9B). Nodal acts through type II receptors and ALK7 to induce TIMP-1 secretion, leading to a decrease in MMP-2/MMP-9 activity. The decrease in MMP activities prevents extracellular matrix degradation, therefore decreasing the invasion of trophoblast cells. Further studies are necessary to identify additional pathways that mediate the anti-invasive action of Nodal and ALK7.

\section{References}

1. Zhou X, Sasaki H, Lowe L, Hogan BL, Kuehn MR: Nodal is a novel TGF- $\beta$-like gene expressed in the mouse node during gastrulation. Nature 1993, 361:543-547

2. Reissmann $\mathrm{E}$, Jornvall $\mathrm{H}$, Blokzijl A, Andersson O, Chang C, Minchiott G, Persico MG, Ibanez CF, Brivanlou AH: The orphan receptor ALK7 and the Activin receptor ALK4 mediate signaling by Nodal proteins during vertebrate development. Genes Dev 2001, 15:2010-2022

3. Bianco C, Adkins HB, Wechselberger C, Seno M, Normanno N, De Luca A, Sun Y, Khan N, Kenney N, Ebert A, Williams KP, Sanicola M, Salomon DS: Cripto-1 activates nodal-and ALK4-dependent and -independent signaling pathways in mammary epithelial cells. Mol Cell Biol 2002 22:2586-2597

4. Brennan J, Norris DP, Robertson EJ: Nodal activity in the node governs left-right asymmetry. Genes Dev 2002, 16:2339-2344

5. Eimon PM, Harland RM: Effects of heterodimerization and proteolytic processing on Derriere and Nodal activity: implications for mesoderm induction in Xenopus. Development 2002, 129:3089-3103

6. Nonaka S, Shiratori H, Saijoh Y, Hamada H: Determination of left-right patterning of the mouse embryo by artificial nodal flow. Nature 2002 , 418:96-99

7. Duboc V, Lapraz F, Saudemont A, Bessodes N, Mekpoh F, Haillot E, Quirin M, Lepage T: Nodal and BMP2/4 pattern the mesoderm and endoderm during development of the sea urchin embryo. Development 2010, 137:223-235

8. Ma GT, Soloveva V, Tzeng SJ, Lowe LA, Pfendler KC, lannaccone PM, Kuehn MR, Linzer DI: Nodal regulates trophoblast differentiation and placental development. Dev Biol 2001, 236:124-135

9. Guzman-Ayala M, Ben-Haim N, Beck S, Constam DB: Nodal protein processing and fibroblast growth factor 4 synergize to maintain a trophoblast stem cell microenvironment. Proc Natl Acad Sci U S A 2004, 101:15656-15660

10. Roberts HJ, Hu S, Qiu Q, Leung PC, Caniggia I, Gruslin A, Tsang B, Peng $C$ : Identification of novel isoforms of activin receptor-like kinase 7 (ALK7) generated by alternative splicing and expression of ALK7 and its ligand, Nodal, in human placenta. Biol Reprod 2003, 68:1719-1726

11. Munir S, Xu G, Wu Y, Yang B, Lala PK, Peng C: Nodal and ALK7 inhibit proliferation and induce apoptosis in human trophoblast cells. J Biol Chem 2004, 279:31277-31286

12. Caniggia I, Mostachfi H, Winter J, Gassmann M, Lye SJ, Kuliszewski M, Post M: Hypoxia-inducible factor-1 mediates the biological effects of oxygen on human trophoblast differentiation through TGF $\beta$ (3). J Clin Invest 2000, 105:577-587

13. Chakraborty C, Gleeson LM, McKinnon T, Lala PK: Regulation of human trophoblast migration and invasiveness. Can J Physiol Pharmacol 2002, 80:116-124

14. Knofler M: Critical growth factors and signalling pathways controlling human trophoblast invasion. Int J Dev Biol 2009, 54:269-280

15. Aplin JD: In vitro analysis of trophoblast invasion. Methods Mol Med 2006, 122:45-57

16. Caniggia I, Winter J, Lye SJ, Post M: Oxygen and placental development during the first trimester: implications for the pathophysiology of pre-eclampsia. Placenta 2000, 21(Suppl A):S25-S30

17. Cross JC: Placental function in development and disease. Reprod Fertil Dev 2006, 18:71-76
18. Noris M, Perico N, Remuzzi G: Mechanisms of disease: Pre-eclampsia. Nat Clin Pract Nephrol 2005, 1:98-114; quiz 120

19. Chelbi ST, Vaiman D: Genetic and epigenetic factors contribute to the onset of preeclampsia. Mol Cell Endocrinol 2008, 282:120-129

20. Caniggia I, Grisaru-Gravnosky S, Kuliszewsky M, Post M, Lye SJ: Inhibition of TGF- $\beta 3$ restores the invasive capability of extravillous trophoblasts in preeclamptic pregnancies. J Clin Invest 1999, 103: 1641-1650

21. Muttukrishna S, North RA, Morris J, Schellenberg JC, Taylor RS, Asselin J, Ledger W, Groome N, Redman CW: Serum inhibin A and activin $A$ are elevated prior to the onset of pre-eclampsia. Hum Reprod 2000, 15:1640-1645

22. Venkatesha S, Toporsian M, Lam C, Hanai J, Mammoto T, Kim YM, Bdolah Y, Lim KH, Yuan HT, Libermann TA, Stillman IE, Roberts D, D'Amore PA, Epstein FH, Sellke FW, Romero R, Sukhatme VP, Letarte M, Karumanchi SA: Soluble endoglin contributes to the pathogenesis of preeclampsia. Nat Med 2006, 12:642-649

23. Wang A, Rana S, Karumanchi SA: Preeclampsia: the role of angiogenic factors in its pathogenesis. Physiology (Bethesda) 2009, 24 147-158

24. Moses EK, Fitzpatrick E, Freed KA, Dyer TD, Forrest S, Elliott K, Johnson MP, Blangero J, Brennecke SP: Objective prioritization of positional candidate genes at a quantitative trait locus for pre-eclampsia on 2q22. Mol Hum Reprod 2006, 12:505-512

25. Roten LT, Johnson MP, Forsmo S, Fitzpatrick E, Dyer TD, Brennecke SP, Blangero J, Moses EK, Austgulen R: Association between the candidate susceptibility gene ACVR2A on chromosome $2 q 22$ and pre-eclampsia in a large Norwegian population-based study (the HUNT study). Eur J Hum Genet 2009, 17:250-257

26. Graham CH, Hawley TS, Hawley RG, MacDougall JR, Kerbel RS, Khoo N, Lala PK: Establishment and characterization of first trimester human trophoblast cells with extended lifespan. Exp Cell Res 1993, 206:204-211

27. Baczyk D, Drewlo S, Proctor L, Dunk C, Lye S, Kingdom J: Glial cell missing-1 transcription factor is required for the differentiation of the human trophoblast. Cell Death Differ 2009, 16:719-727

28. Genbacev O, Schubach SA, Miller RK: Villous culture of first trimester human placenta-model to study extravillous trophoblast (EVT) differentiation. Placenta 1992, 13:439-461

29. Nishimura T, Dunk C, Lu Y, Feng X, Gellhaus A, Winterhager E, Rossant J, Lye SJ: Gap junctions are required for trophoblast proliferation in early human placental development. Placenta 2004, 25 595-607

30. Xu G, Zhong Y, Munir S, Yang BB, Tsang BK, Peng C: Nodal induces apoptosis and inhibits proliferation in human epithelial ovarian cancer cells via activin receptor-like kinase 7. J Clin Endocrinol Metab 2004 89:5523-5534

31. Khoo NK, Bechberger JF, Shepherd T, Bond SL, McCrae KR, Hamilton GS, Lala PK: SV40 Tag transformation of the normal invasive trophoblast results in a premalignant phenotype, I: mechanisms responsible for hyperinvasiveness and resistance to anti-invasive action of TGF $\beta$. Int J Cancer 1998, 77:429-439

32. Morrish DW, Whitley GSJ, Cartwright JE, Graham $\mathrm{CH}$, Caniggia I: In vitro models to study trophoblast function and dysfunction: a workshop report. Placenta 2002, 23:S114-S118

33. Kilburn BA, Wang J, Duniec-Dmuchowski ZM, Leach RE, Romero R, Armant DR: Extracellular matrix composition and hypoxia regulate the expression of HLA-G and integrins in a human trophoblast cell line. Biol Reprod 2000, 62:739-747

34. Chirco R, Liu XW, Jung KK, Kim HR: Novel functions of TIMPs in cell signaling. Cancer Metastasis Rev 2006, 25:99-113

35. Huppertz B, Frank HG, Reister F, Kingdom J, Korr H, Kaufmann P Apoptosis cascade progresses during turnover of human trophoblast: analysis of villous cytotrophoblast and syncytial fragments in vitro. Lab Invest 1999, 79:1687-1702

36. Genbacev O, Joslin R, Damsky CH, Polliotti BM, Fisher SJ: Hypoxia alters early gestation human cytotrophoblast differentiation/invasion in vitro and models the placental defects that occur in preeclampsia J Clin Invest 1996, 97:540-550

37. Genbacev O, Zhou Y, Ludlow JW, Fisher SJ: Regulation of human placental development by oxygen tension. Science 1997, 277:1669-1672

38. Nishi H, Nakada T, Hokamura M, Osakabe Y, Itokazu O, Huang LE, Isaka K: Hypoxia-inducible factor-1 transactivates transforming growth factor- $\beta 3$ in trophoblast. Endocrinology 2004, 145:4113-4118 
Nodal Inhibits Trophoblast Invasion 1189

AJP March 2011, Vol. 178, No. 3

39. Harris LK: Review: trophoblast-vascular cell interactions in early pregnancy: how to remodel a vessel. Placenta 2010, 31(Suppl):S93-S98

40. Anin SA, Vince G, Quenby S: Trophoblast invasion. Hum Fertil (Camb) 2004, 7:169-174

41. Caniggia I, Lye SJ, Cross JC: Activin is a local regulator of human cytotrophoblast cell differentiation. Endocrinology 1997, 138: 3976-3986
42. Cohen M, Bischof P: Factors regulating trophoblast invasion. Gynecol Obstet Invest 2007, 64:126-130

43. Huppertz B, Kadyrov M, Kingdom JC: Apoptosis and its role in the trophoblast. Am J Obstet Gynecol 2006, 195:29-39

44. Myers JE, Merchant SJ, Macleod M, Mires GJ, Baker PN, Davidge ST: MMP-2 levels are elevated in the plasma of women who subsequently develop preeclampsia. Hypertens Pregnancy 2005, 24:103-115 